\title{
Positioning features of vocal, semantic, and graphemic task zones in Chinese-English bilinguals
}

\author{
Han Gao ${ }^{1}$, Zhisheng Chen ${ }^{1}$, Weixiong Zhuang ${ }^{2}$, Zhihe Chen ${ }^{1}$, Shaotao Tan ${ }^{1}$, Xichang Lan ${ }^{1}$, Shikun Lian ${ }^{1}$, \\ Xuying $\mathrm{He}^{3}$ \\ ${ }^{1}$ Department of Neurosurgery, ${ }^{2}$ Department of Radiology, Yunfu People's Hospital, Yunfu 527300, China; ${ }^{3}$ Department of Neurosurgery, Zhujiang \\ Hospital, Southern Medical University, Guangzhou 510282, China \\ Contributions: (I) Conception and design: H Gao, X He; (II) Administrative support: Z Chen, W Zhuang, Z Chen, S Tan, X Lan, S Lian; (III) \\ Provision of study materials or patients: All authors; (IV) Collection and assembly of data: H Gao, X He; (V) Data analysis and interpretation: H \\ Gao, X He; (VI) Manuscript writing: All authors; (VII) Final approval of manuscript: All authors. \\ Correspondence to: Xuying He. Department of Neurosurgery, Zhujiang Hospital, Southern Medical University, Guangzhou 510282, China. \\ Email: 2517079319@qq.com.
}

Background: Bilinguals are people that can use two languages for oral communication. Many bilinguals have specific language-associated cortical regions. This study aimed to analyze the cortical positioning features of vocal, semantic, and graphemic task zones in unskilled late Chinese (first)-English (second) (ULCE) bilinguals using the blood-oxygen-level dependent functional magnetic resonance imaging (BOLDfMRI) technique.

Methods: Twelve ULCE bilinguals were assigned Chinese-English (C-E) vocal, semantic, and graphemic tasks; SPM8 software was used to compare and investigate the brain activation maps towards different language tasks and to calculate their corresponding lateralization indexes (LIs).

Results: These three language tasks in simple Chinese could activate most traditional language zones, which all exhibited obvious left-deviated activation dominance. A simple English task could also activate most traditional language zones, but only the semantic task appeared to have obvious left-deviated lateralization. However, none of the three tasks displayed any specific language zones between Chinese and English on the group level.

Conclusions: Vocal, semantic, and graphemic tasks can all activate multiple brain regions in the language network and reflect their respective cognitive processing characteristics. Bilingual processing in ULCE bilinguals has similar neural mechanisms, but the left-deviated lateralization is not obvious when performing English vocal and graphemic tasks.

Keywords: Bilinguals; cortical region; task; magnetic resonance imaging (MRI)

Submitted Oct 16, 2019. Accepted for publication Oct 21, 2019.

doi: 10.21037/atm.2019.10.119

View this article at: http://dx.doi.org/10.21037/atm.2019.10.119

\section{Introduction}

Bilinguals are people that can use two languages for communication (1-3). Bilinguals can be divided into early and late types according to whether they had contact with their second language before or after the age of 5 (4). They can also be divided into skilled and unskilled types according to the level of proficiency gained in their two languages. A skilled bilingual has similar proficiencies in both languages, while the second language of an unskilled bilingual cannot reach a similar proficiency as his/ her native language (5). Kim et al. (6) applied the silent sentence-generation task to assess bilingual performance and found that the language areas in the temporal lobe of early or late bilinguals essentially overlapped or only 
showed a subtle difference. However, the areas in the frontal lobe in late bilinguals had clear separation, while no difference was found above the frontal lobe areas in early bilinguals. The direct cortical electrical stimulation (DCES) technique is the gold standard in testing language areas. Many studies have demonstrated the presence of specific cortical language areas in bilinguals using DCES; specifically, they found that the electrical stimulation on certain cortical areas caused disturbances in only one of the two languages (7-10). This shows that the language areas of two languages may not fully overlapped. Chee et al. (11) applied the vision-presenting reading task to compare the blood-oxygen-level dependent functional magnetic resonance imaging (BOLD-fMRI) activation zones of the Chinese and English languages in skilled bilinguals. They discovered that these bilinguals used the common semantic analysis system when implementing Chinese and English tasks. Therefore, it was concluded that the bilinguals with Chinese as their native language used similar neural mechanisms when implementing Chinese and English tasks. Pu et al. (12) also implemented the vision-presenting verb generation task to study the representative zones of Chinese and English in unskilled bilinguals. They determined that bilinguals with Chinese as their native language also used similar neural mechanisms when implementing Chinese and English processing tasks. Xue et al. (13) carried out the BOLD-fMRI study in late Chinese-English (C-E) bilinguals using semantic decision tasks and found no specific activation zone in these bilinguals in the C-E semantic level; in other words, they concluded that the representative zones of these two languages are basically the same. Taken together, the results outlined above concerning unskilled bilinguals or late bilinguals are still controversial, and it remains unclear whether there is a separation between the representative zones of the two languages. The above studies only performed testing in the semantic domain; however, a language can generally be divided into the four aspects: semantics, voice, grapheme, and syntax. Therefore, this study was designed to further add the vocal and graphemic tasks based on the above studies. The objective was to determine the distribution characteristics of BOLDfMRI language zones in unskilled late Chinese (first)English (second) (ULCE) bilinguals when executing semantic, vocal, and graphemic tasks, and to judge whether these ULCE bilinguals used the same or different neural mechanisms when performing different language tasks.

\section{Methods}

\section{Subjects}

Twelve healthy volunteers ( 6 males and 6 females, aged 21 to 24 years, mean 22.3 years old) were enrolled in this study. They were all the senior students majoring in English in Guangdong University of Foreign Studies, with normal vision or corrected vision and Mandarin as their native language. None of the subjects had a history of neurological disease. All of them were right-handed as determined by the Edinburgh handedness inventory (EHI). Furthermore, their mini-mental state examination (MMSE) scores were all about 30 points. MRI brain scanning revealed no obvious abnormality, and they all voluntarily agreed to participate in the study.

\section{Experimental tasks}

The language stimulation programs were as follows: the semantics, vocal, and graphitic judgment tasks were performed, and all blocks of the tasks were designed and visually presented using the E-Prime software (Psychology Software Tools Inc., CA, USA). Each subject completed two trail task scans designed by Latin square. The experimental pictures were screened from a picture library; all the pictures were black-white pictures of the same size. The experimental Chinese and English words were the commonly used words selected from Chinese and English word frequency tables, respectively (Figure 1).

\section{Experimental task 1 (semantic task)}

The objective of the task was to judge whether the two presented Chinese or English words were the same part of speech: namely, whether they were both abstract words or entity words (consistent), or one abstract word and one entity word (inconsistent). The Chinese and English tasks appeared alternately in each block.

\section{Experimental task 2 (vocal task)}

The objective of the Chinese judgment task was to determine whether the two Chinese words had a consistent tone of voice: namely, whether the two test words had the same tone (consistent), or a different tone (inconsistent). In order to simplify the assessment, only the second (rising tone) and fourth tones (falling tone) were selected.

The objective of the English judgment task was to determine whether the accented syllables of two English words were consistent: namely, whether both test words 

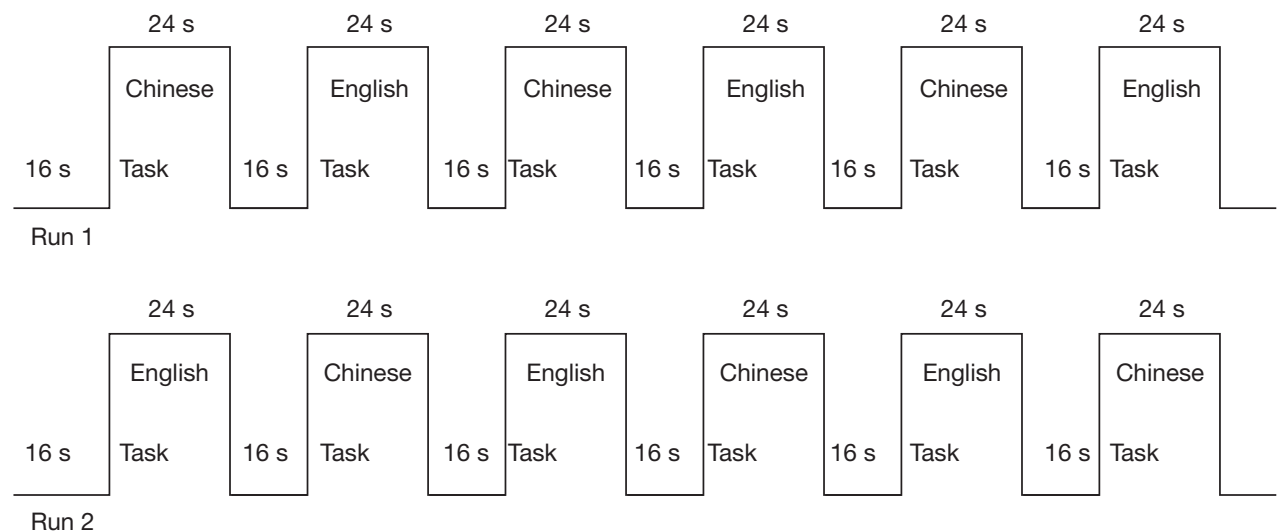

Figure 1 Task flow sheets.

were accented on the same (first or second) syllable, or whether they were accented on different syllables (inconsistent). In order to simplify the assessment, words with only two syllables were selected. The Chinese and English tasks appeared alternately in each block.

\section{Experimental task 3 (graphemic task)}

The objective of the Chinese judgment task was to determine whether the presented Chinese character was a real character; the objective of the English judgment task was to determine whether the presented English word was a real word. The Chinese and English tasks appeared alternately in each block.

The baseline tasks of all the above tasks used the same prompt words with the same lumen on the visual screen; during the scanning process, the right and left buttons were used to determine "consistent" or "inconsistent", respectively. Before the study, all the subjects were trained and had their behavioral data collected; the accuracy rates of each Chinese and English task were between 98-100\% and were similar $(\mathrm{P}=0.343)$.

\section{Data collection and analysis}

A 12-coil Siemens $1.5 \mathrm{~T}$ superconductive MRI system was used for the scanning, and SPM8 (Wellcome Department of Imaging Neuroscience at University College London) was used for the analysis. The activation maps were displayed with SPM8-owned MNI coordinates with the activated regions $\geq 10$ adjacent voxels as the activation standard [family-wise error (FWE) correction, $\mathrm{P}<0.001$ ]. The XJview 8 (http://www.alivelearn.net/xjview/) plug-in was used to convert the MNI coordinates of the activated regions into the anatomical locations and automatically mark the anatomical position of the activated clump center.

\section{Calculation of lateralization index (LI)}

The $\mathrm{LI}$ was calculated as follows: $\mathrm{LI}=(\mathrm{L}-\mathrm{R}) /(\mathrm{L}+\mathrm{R})[\mathrm{L}$ : activated voxels in the region of interest (ROI) of the left hemisphere; R: activated voxels in the ROI of right hemisphere; LI $>0.1$, left dominance], ROI selected bilateral middle frontal gyrus, inferior frontal gyrus, superior temporal gyrus, middle temporal gyrus, superior parietal lobule, and inferior parietal lobule.

\section{Results}

\section{Activation areas of the Chinese semantic task}

As shown in Figures 2,3, the language areas for the simple Chinese semantic task (FWE correction, $\mathrm{P}<0.001$, voxel $\geq 10$ ) were as follows: right inferior frontal gyrus, right middle frontal gyrus, right precentral gyrus, right anterior cingulate gyrus, right postcentral gyrus, right inferior parietal lobule, right insular lobe, right precuneus, right parahippocampal gyrus, right lingual gyrus, right thalamus, right cerebellum, left medial frontal gyrus, left middle frontal gyrus, left inferior frontal gyrus, left anterior cingulate gyrus, left cingulate gyrus, left precentral gyrus, left postcentral gyrus, left inferior parietal lobule, left insular lobe, left thalamus, and left caudate nucleus.

\section{Activation areas of the English semantic task}

The language areas for the simple English semantic task 


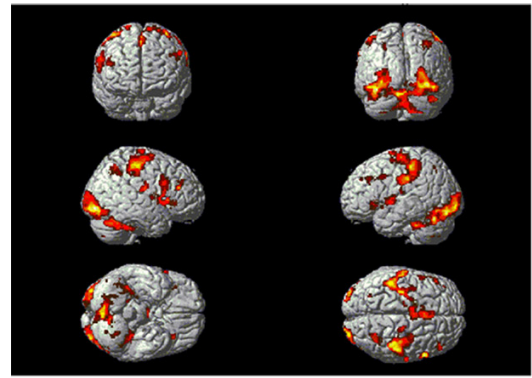

Semantic task

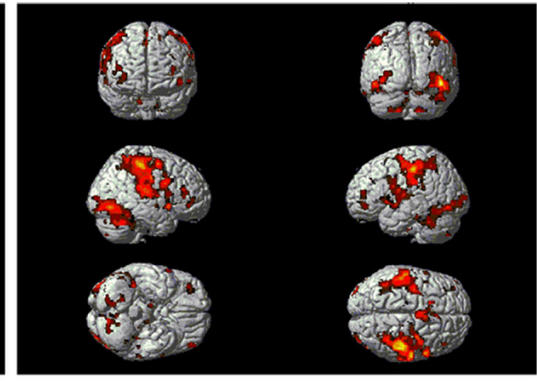

Vocal task

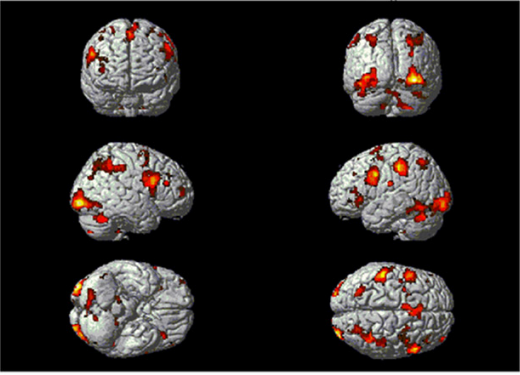

Graphemic task

Figure 2 Simple Chinese task-activated language areas.

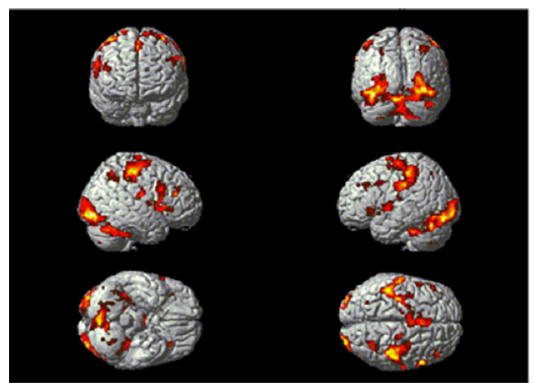

Semantic task

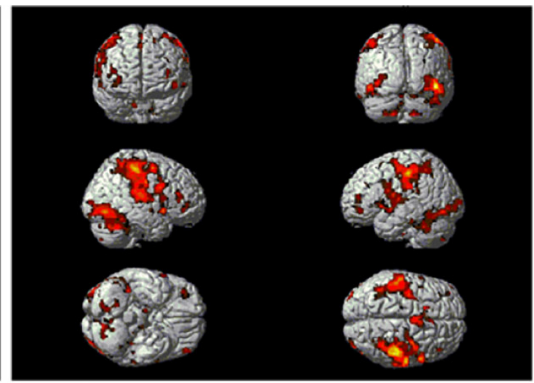

Vocal task

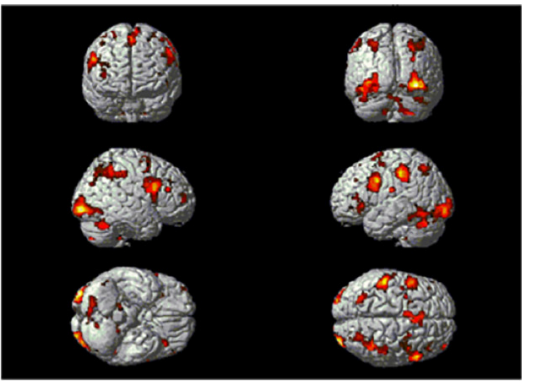

Graphemic task

Figure 3 Simple English task-activated language areas.

[FWE correction, $\mathrm{P}<0.001$, voxel $\geq 10$ ] were as follows: right insular lobe, right middle frontal gyrus, right inferior frontal gyrus, right precentral gyrus, right postcentral gyrus, right inferior parietal lobule, right cingulate gyrus, right cerebellum, right precuneus, right thalamus, right caudate nucleus, left insular lobe, left middle frontal gyrus, left precentral gyrus, left superior temporal gyrus, left inferior parietal lobule, left cingulate gyrus, left anterior cingulate gyrus, left thalamus, and left precuneus.

\section{Activation areas of the Chinese phonological task}

The language areas for simple Chinese phonological task (FWE correction, $\mathrm{P}<0.001$, voxel $\geq 10$ ) were as follows: right middle frontal gyrus, right lateral angular gyrus, right superior parietal lobule, right inferior parietal lobule, right cingulate gyrus, right posterior cingulate gyrus, right parahippocampal gyrus, right postcentral gyrus, right transverse temporal gyrus, right cerebellum, right thalamus, right lentiform nucleus, right precuneus, right fusiform gyrus, right inferior occipital gyrus, left medial frontal gyrus, left middle frontal back, left inferior frontal gyrus, left insular lobe, left middle temporal gyrus, left transverse temporal gyrus, left cingulate gyrus, left anterior cingulate gyrus, left precentral gyrus, left postcentral gyrus, left inferior parietal lobule, left supramarginal gyrus, left cerebellum, left lentiform nucleus, left thalamus, left cuneus, left precuneus, and left caudate nucleus.

\section{Activation areas of the English phonological task}

The language areas for the simple English phonological task (FWE correction, $\mathrm{P}<0.001$, voxel $\geq 10$ ) were as follows: right precentral gyrus, right inferior parietal lobule, right insular lobe, right fusiform gyrus, right cerebellum, right middle occipital gyrus, right medial frontal gyrus, right middle frontal gyrus, right superior temporal gyrus, right caudate nucleus, right thalamus, right posterior cingulate gyrus, right postcentral gyrus, right precuneus, right parahippocampal gyrus, left precentral gyrus, left inferior parietal lobule, left cuneus, left insular lobe, left claustrum, left medial frontal gyrus, left superior frontal gyrus, left caudate nucleus, left thalamus, left inferior frontal gyrus, left lentiform nucleus, left cingulate gyrus, left lateral 
Table 1 LIs of different simple Chinese or English language tasks

\begin{tabular}{|c|c|c|c|c|c|c|}
\hline Area & \multicolumn{2}{|c|}{ Semantic task } & \multicolumn{2}{|c|}{ Phonological task } & \multicolumn{2}{|c|}{ Morphological task } \\
\hline Frontal lobe & 0.36 & 0.34 & 0.13 & -0.26 & 0.27 & -0.16 \\
\hline Temporal lobe & 0.78 & 0.45 & 0.23 & -0.35 & 0.43 & 0.07 \\
\hline
\end{tabular}

LI, lateralization index.

angular gyrus, left lingual gyrus, and left middle frontal gyrus.

\section{Activation areas of the Chinese morphological task}

The language areas for the simple Chinese morphological task (FWE correction, $\mathrm{P}<0.001$, voxel $\geq 10$ ) were as follows: right medial frontal gyrus, right fusiform gyrus, right inferior occipital gyrus, right middle occipital gyrus, right postcentral gyrus, right inferior parietal lobule, right claustrum, right precentral gyrus, right inferior frontal gyrus, right cerebellum, right insular lobe, right superior parietal lobule, right cingulate gyrus, right thalamus, left postcentral gyrus, left inferior parietal lobule, left superior frontal gyrus, left precentral gyrus, left middle frontal gyrus, left middle occipital gyrus, left superior parietal lobule, and left insular lobe.

\section{Activation areas of the English morphological task}

The language areas for the simple English morphological task (FWE correction, $\mathrm{P}<0.001$, voxel $\geq 10$ ) were as follows: right inferior occipital gyrus, right fusiform gyrus, right cingulate gyrus, right inferior parietal lobule, right precuneus, right middle frontal gyrus, right insular lobe, right inferior frontal gyrus, right cerebellum, right precentral gyrus, right claustrum, left supramarginal gyrus, left inferior parietal lobule, left superior frontal gyrus, left insular lobe, left precentral gyrus, left inferior frontal gyrus, left cuneus, left cerebellum, left cingulate gyrus, left posterior cingulate gyrus, left superior parietal lobule, left precuneus, left middle frontal gyrus, left precentral gyrus, and left inferior frontal gyrus.

\section{LIs of different language tasks}

As shown in Table 1, the LIs of the semantic task for Chinese were 0.36 (frontal lobe) and 0.78 (temporal lobe), and those for English were 0.34 (frontal lobe) and 0.45 (temporal lobe). The LIs of the phonological task for Chinese were 0.13 (frontal lobe) and 0.23 (temporal lobe), and those for English were -0.26 (frontal lobe) and -0.35 (temporal lobe). The LIs of the morphological task for Chinese were 0.27 (frontal lobe) and 0.43 (temporal lobe), and those for English were -0.16 (frontal lobe) and 0.07 (temporal lobe).

\section{Specific language areas}

The group comparison of C-E-mixed tasks (FWE correction, $\mathrm{P}<0.05$, voxel $\geq 10$ ) showed that all three language tasks had no specifically activated brain area.

\section{Discussion}

Currently, studies about C-E bilinguals in China and abroad are mainly focused on skilled or early bilinguals; however, research targeting ULCE bilinguals is rare, so it is clinically urgent to understand the language zone distributions in these bilinguals. Most scholars believe that under the resolution provided by BOLD-fMRI, the representative zones of the first and second languages in skilled and early bilinguals are substantially coincident. Although the second language might lead to greater activation intensity, this is presumed to only be caused by the greater computational needs rather than the different representative zones of the second language and the native language (14). Whether representative bilingual zones in unskilled or late bilinguals exist separately is still controversial. Kim et al. (6) believe that differences exist. Meanwhile, Rapport et al. (7), Roux et al. (8), Bello et al. (10), and Giussani et al. (5) all performed wake-up surgery plus direct cortical electric stimulation and found that specific bilingual areas, specifically cortical language areas, were present and not entirely coincident. In this study, ULCE bilinguals all performed vocal, semantic, and graphemic tasks, but the BOLD-fMRI analysis did not find any specific language areas in these bilinguals. In other words, the representative 
zones of these two languages in the cortex were similar, so the brain might have the same neural mechanism when processing these two languages.

Previous bilingual BOLD-fMRI studies mainly implemented semantic task or a mixture of tasks of spontaneous language; taking into account that a language is composed of voice, semantics, graphemes, and syntax, research targeting each individual item might help to further understand the neural mechanisms of bilinguals. This study was the first study that specifically targeted the cortical representative zones of the vocal and graphemic tasks in ULCE bilinguals, and no specific activated region was found in the vocal and graphemic levels: ULCE bilinguals used similar neural mechanisms in processing vocal and graphemic tasks. Furthermore, it was found that no specific activated region appeared in the semantic level between the two languages, which is similar to previous findings $(12,13)$.

A previous study found that Chinese users exhibited obvious lateralization in semantic, vocal, and graphemic tasks while this lateralization appeared in English users only in semantic tasks; this result might be explained by the fact that the tested subjects were unskilled with the second language (15). When performing language tasks, the leftdeviated lateralization in the BOLD-fMRI results was one method to measure whether this language task could effectively activate the language areas (16). The results of this study showed that for ULCE bilinguals, their left language area was much more obviously activated when performing the bilingual semantic tasks. Mixed bilingual tasks could display C-E activation zones in a single scan, and could thus have an advantage over implementing Chinese and English tasks individually by virtue of reducing the scanning time and co-displaying the bilingual language activation regions. Therefore, a C-E mixed semantic task could be used as the BOLD-fMRI stimulation task for the preoperative language area positioning in the language functional region surgery of ULCE bilinguals.

This study only applies the BOLD-fMRI technique to study the cortical representative zones of Chinese and English, but the limited resolution of this technique might be the reason why no specific cortical representative region was found. Burgaleta et al. (17) compared the subcortical structures of early bilinguals and monolinguals and found significant morphological expansions in the bilateral putamen and thalamus, in addition to the left globus pallidus and right caudate nucleus. Pliatsikas et al. (18) applied MR tractography to study skilled late bilinguals and found as the proficiency of the second language increased, the subjects' white matter structures were affected accordingly. These all indicate that structural brain changes in bilinguals might possibly exist. Because the subjects of this study were ULCE bilinguals, the impacts of these two languages on brain tissue structures, particularly the subcortical structures, might still be too minor to be detected by BOLD-fMRI.

\section{Acknowledgments}

Funding: This work was supported by the Natural Science Foundation of Guangdong Province, China (No. 2014A030310001).

\section{Footnote}

Conflicts of Interest: The authors have no conflicts of interest to declare.

Ethical Statement: The authors are accountable for all aspects of the work in ensuring that questions related to the accuracy or integrity of any part of the work are appropriately investigated and resolved. The research protocol was previously approved by the Ethical Committee of the Yunfu People's Hospital (No. 2019A001).

\section{References}

1. Gómez-Ruiz MI. Bilingualism and the brain: myth and reality. Neurologia 2010;25:443-52.

2. Alladi $S, B a k T H$, Mekala $S$, et al. Impact of bilingualism on cognitive outcome after stroke. Stroke 2016;47:258-61.

3. Bak TH, Alladi S. Bilingualism, dementia and the tale of many variables: why we need to move beyond the Western World. Commentary on Lawton et al. (2015) and FullerThomson (2015). Cortex 2016;74:315-7.

4. Fabbro F. The bilingual brain: cerebral representation of languages. Brain Lang 2001;79:211-22.

5. Giussani C, Roux FE, Lubrano V, et al. Review of language organisation in bilingual patients: what can we learn from direct brain mapping? Acta Neurochir (Wien) 2007;149:1109-16; discussion 1116.

6. Kim KH, Relkin NR, Lee KM, et al. Distinct cortical areas associated with native and second languages. Nature 1997;388:171-4.

7. Rapport RL, Tan CT, Whitaker HA. Language function and dysfunction among Chinese- and English-speaking polyglots: cortical stimulation, Wada testing, and clinical 
studies. Brain Lang 1983;18:342-66.

8. Roux FE, Lubrano V, Lauwers-Cances V, et al. Intraoperative mapping of cortical areas involved in reading in mono- and bilingual patients. Brain 2004;127:1796-810.

9. Högl B. What the "man in the moon" can tell us about the future of our brains. Ann Transl Med 2017;5:358.

10. Bello L, Acerbi F, Giussani C, et al. Intraoperative language localization in multilingual patients with gliomas. Neurosurgery 2006;59:115-25; discussion 115-25.

11. Chee MW, Caplan D, Soon CS, et al. Processing of visually presented sentences in Mandarin and English studied with fMRI. Neuron 1999;23:127-37.

12. $\mathrm{Pu}$ Y, Liu HL, Spinks JA, et al. Cerebral hemodynamic response in Chinese (first) and English (second) language processing revealed by event-related functional MRI. Magn Reson Imaging 2001;19:643-7.

13. Xue G, Dong Q, Jin Z, et al. Mapping of verbal working memory in nonfluent Chinese-English bilinguals with functional MRI. Neuroimage 2004;22:1-10.

Cite this article as: Gao H, Chen Z, Zhuang W, Chen Z, Tan S, Lan X, Lian S, He X. Positioning features of vocal, semantic, and graphemic task zones in Chinese-English bilinguals. Ann Transl Med 2019;7(22):616. doi: 10.21037/atm.2019.10.119
14. Luke KK, Liu HL, Wai YY, et al. Functional anatomy of syntactic and semantic processing in language comprehension. Hum Brain Mapp 2002;16:133-45.

15. Breshears JD, Gaona CM, Roland JL, et al. Mapping sensorimotor cortex with slow cortical potential restingstate networks while awake and under anesthesia. Neurosurgery 2012;71:305-16; discussion 316.

16. Petrovich Brennan NM, Whalen S, de Morales Branco D, et al. Object naming is a more sensitive measure of speech localization than number counting: converging evidence from direct cortical stimulation and fMRI. Neuroimage 2007;37 Suppl 1:S100-8.

17. Burgaleta $M$, Sanjuán A, Ventura-Campos N, et al. Bilingualism at the core of the brain. Structural differences between bilinguals and monolinguals revealed by subcortical shape analysis. Neuroimage 2016;125:437-45.

18. Pliatsikas C, Moschopoulou E, Saddy JD. The effects of bilingualism on the white matter structure of the brain. Proc Natl Acad Sci U S A 2015;112:1334-7. 